\title{
Strong Convergence of a New Hybrid Algorithm for Fixed Point Problems and Equilibrium Problems
}

\section{Dang Van Hieu}

Applied Analysis Research Group, Faculty of Mathematics and Statistics, Ton Duc Thang University

Ho Chi Minh City, Vietnam

E-mail(corresp.): dangvanhieu@tdtu.edu.vn

Received October 16, 2017; revised October 10, 2018; accepted October 11, 2018

\begin{abstract}
The paper considers the problem of finding a common solution of a pseudomonotone and Lipschitz-type equilibrium problem and a fixed point problem for a quasi nonexpansive mapping in a Hilbert space. A new hybrid algorithm is introduced for approximating a solution of this problem. The presented algorithm can be considered as a combination of the extragradient method (two-step proximallike method) and a modified version of the normal Mann iteration. It is well known that the normal Mann iteration has the weak convergence, but in this paper we has obtained the strong convergence of the new algorithm under some mild conditions on parameters. Several numerical experiments are reported to illustrate the convergence of the algorithm and also to show the advantages of it over existing methods.
\end{abstract}

Keywords: proximal-like method, extragradient method, equilibrium problem, fixed point problem.

AMS Subject Classification: 65J15; 47H05; 47J25; 47J20; 91B50.

\section{Introduction}

The equilibrium problem (EP) can be considered as a general model in the sense that it includes, as special cases, many mathematical models such as variational inequality problems, optimization problems, fixed point problems, complementarity problems and Nash equilibrium problems, see, e.g., $[5,8,18$, 30]. Mathematically, this (EP) problem can be stated as follows:

$$
\text { Find } x^{*} \in C \text { such that } f\left(x^{*}, y\right) \geq 0, \forall y \in C \text {, }
$$

Copyright (c) 2019 The Author(s). Published by VGTU Press

This is an Open Access article distributed under the terms of the Creative Commons Attribution License (http://creativecommons.org/licenses/by/4.0/), which permits unrestricted use, distribution, and reproduction in any medium, provided the original author and source are credited. 
where $C$ is a nonempty closed convex subset of a real Hilbert space $H$ and $f: H \times H \rightarrow \Re$ is a given bifunction with $f(x, x)=0$ for all $x \in C$. We denote $E P(f, C)$ the solution set of problem (1.1). Problem (1.1) is also well known as the Ky Fan inequality early studied in [9]. In recent years, this problem has received a lot of attention by many authors, for instance, see $[1,2,6,10,11,12,13,14,15,17,23,26,27,28,32]$. This interest can be that, as be seen, it unifies all the aforementioned particular problems in a convenient way.

One of the most popular methods for solving problem (1.1) is the proximal point method $[17,28]$. The main idea of this method is to replace the original problem by a family of regularized equilibrium problems which can be solved more easily. Regularized solutions can finitely or asymptotically converge to some solution of the original problem. Another notable method is the proximal-like method presented in [10]. This method was further extended and investigated the convergence by the authors in [32] under different assumptions that the cost bifunctions are pseudomonotone and satisfy the Lipschitz-type condition [26]. The methods in [10,32] are also called extragradient methods (or two-step proximal-like methods) due to the results obtained by Korpelevich in [19] for saddle problems. The advantages of the extragradient methods in $[10,32]$ are that they are used for the class of pseudomonotone bifunctions and can be easier to numerically solve than the proximal point method in $[17,28]$.

In this paper, we are interested in the problem of finding a common solution of an equilibrium problem and a fixed point problem in a Hilbert space [15, $20,21,24,25,31,35,36,37]$. The motivation and inspiration for studying such this common solution problem is in its possible application to mathematical models whose constraints can be expressed as fixed point problems. This is the case, in particular, in practical problems as signal processing, network resource allocation and image recovery; see, for instance, [16,24,25]. Let $U: H \rightarrow H$ is a mapping, the fixed point problem (FPP) for the mapping $U$ is to find $x^{*} \in H$ such that

$$
U x^{*}=x^{*} .
$$

The solution set of problem (1.2) is called the fixed point set of $U$ and is denoted by Fix $(U)$. Most of the methods for solving problem (1.2) are obtained from the normal Mann iteration, namely, from $x_{0} \in H$, compute $x_{n+1}$ for all $n \geq 0$ by

$$
x_{n+1}=\alpha_{n} x_{n}+\left(1-\alpha_{n}\right) U x_{n},
$$

where the parameter sequence $\left\{\alpha_{n}\right\}$ has to satisfy some properties to take the weak convergence. In order to get the strong convergence, another regularized iterative method, which is more useful in infinite dimensional Hilbert spaces, is the Halpern iteration, namely

$$
x_{n+1}=\alpha_{n} x_{0}+\left(1-\alpha_{n}\right) U x_{n},
$$

where $x_{0} \in H$ and the sequence $\left\{\alpha_{n}\right\} \subset(0,1)$ is slowly diminishing and nonsummable, i.e., $\alpha_{n} \rightarrow 0$ and $\sum_{n=0}^{\infty} \alpha_{n}=+\infty$. Besides, it is worth noting that, together with the Halpern iteration is the general form of it, namely the viscosity method [29], in which the cost mapping $U$ is incorporated with a 
contraction mapping in the iterates. Finally, another method, which also provides the strong convergence, is the hybrid steepest descent method introduced in $[39]$.

In 2007, for finding a common solution of a problem (1.1) and a problem (1.2), Takahashi and Takahashi [35] introduced the viscosity approximation method where the authors have combined the proximal point method $[17,28]$ with the viscosity technique [29]. Under some suitable conditions imposed the control parameters, the strong convergence of the method was established. In 2008, using a variant of the hybrid steepest descent method for fixed point problems in [39], Maingé and Moudafi [25] proposed an iterative process for finding an element in $\Omega:=E P(f, C) \cap F i x(U)$. The norm convergence of the algorithm has been proved. The algorithm of Maingé and Moudafi in [25] has first improved and developed the results in this field. Another advantage of that algorithm is that it allows to relax substantially the conditions on parameters due to the relaxation process induced on the mapping $U$.

Recently, by using the extragradient method [10,32] and the hybrid steepest descent technique in [39], Vuong et al. [37] have introduced a new numerical algorithm, namely the extragradient viscosity method, for solving an equilibrium problem involving a fixed point problem for a demicontractive mapping. Under assumptions that the bifunction is pseudomonotone and satisfies the Lipschitz-type condition [26], the authors have established the strong convergence of the algorithm. As remarked in [37], this method has the advantage in numerically computing by using the tools in optimization. The extragradient viscosity method in [37] also allows to reduce several strong conditions in establishing the convergence of previously known extragradient algorithms. It is also worth mentioning additionally that other strongly convergent algorithms for finding an element in $\Omega:=E P(f, C) \cap F i x(U)$, which combine the extragradient method with the hybrid or shrinking projection technique, can be found in $[15,31,34,36]$.

In this paper, motivated and inspirated by the results of Takahashi and Takahashi in [35], of Maingé and Moudafi in [25], and of Vuong et al. in [37] and based on the work [22], we introduce a different strongly convergent algorithm as the combination between the extragradient method (two-step proximal-like method) and the Mann-like iteration [7,22] for approximating a common solution of a pseudomonotone and Lipschitz-type equilibrium problem and a fixed point problem for a quasi-nonexpansive mapping. As mentioned below, by considering a relaxation of a demicontractive mapping, then the result in this paper is still true for the more general class of demicontractive mappings. It is emphasized that the normal Mann iteration provides the weak convergence while the algorithm in this paper, which uses the similar version to the Mann iteration, has provided the strong convergence. This is particularly interesting in infinite dimensional Hilbert spaces where the norm convergence is more useful than the weak one. Several of our numerical experiments in both finite and infinite dimensional Hilbert spaces have shown that the new algorithm is promising and has competitive advantage over existing methods.

The remainder of this paper is organized as follows: In Section 2 we collect some definitions and preliminary results used in the paper. Section 3 deals 
with proposing the new algorithm and analyzing its convergence. Finally, in Section 4 we provide several examples to illustrate the numerical behavior of the new algorithm in comparison with the behaviors of previously known algorithms.

\section{Preliminaries}

Let $H$ be a real Hilbert space and $C$ be a nonempty closed convex subset of $H$. It was known that the iterative methods for solving problem (1.1) are often relative to monotone bifunction or operator theory. Now, we review some concepts of monotonicity of a bifunction, see $[5,30]$ for more details. A bifunction $f: H \times H \rightarrow \Re$ is said to be:

- (i) strongly monotone on $C$ if there exists a constant $\gamma>0$ such that

$$
f(x, y)+f(y, x) \leq-\gamma\|x-y\|^{2}, \forall x, y \in C ;
$$

- (ii) monotone on $C$ if

$$
f(x, y)+f(y, x) \leq 0, \forall x, y \in C
$$

- (iii) pseudomonotone on $C$ if

$$
f(x, y) \geq 0 \Longrightarrow f(y, x) \leq 0, \forall x, y \in C ;
$$

- (iv) strongly pseudomonotone on $C$ if there exists a constant $\gamma>0$ such that

$$
f(x, y) \geq 0 \Longrightarrow f(y, x) \leq-\gamma\|x-y\|^{2}, \forall x, y \in C .
$$

From the above definitions, it is easy to see that the following implications hold,

$$
\text { (i) } \Longrightarrow \text { (ii) } \Longrightarrow(\text { iii) and (i) } \Longrightarrow \text { (iv) } \Longrightarrow \text { (iii). }
$$

The converses are not true in general. We say that a bifunction $f: H \times H \rightarrow \Re$ satisfies a Lipschitz-type condition [26] on $H$ if there exist two positive constants $c_{1}, c_{2}$ such that

$$
f(x, y)+f(y, z) \geq f(x, z)-c_{1}\|x-y\|^{2}-c_{2}\|y-z\|^{2}, \forall x, y, z \in H .
$$

Note that if $A: H \rightarrow H$ is a Lipschitz continuous operator, i.e., there exists $L>$ 0 such that $\|A x-A y\| \leq L\|x-y\|$ for all $x, y \in H$, then the bifunction $f(x, y)=$ $\langle A x, y-x\rangle$ satisfies the Lipschitz-type condition with $c_{1}=\frac{L}{2 \mu}$ and $c_{2}=\frac{L \mu}{2}$ for any $\mu>0$. The Lipschitz-type condition is often used in establishing the convergence of extragradient-like methods (two-step proximal-like methods) for EPs (see, e.g., [11, 13, 15, 23, 32,34]).

Let $U: H \rightarrow H$ be a mapping with the fixed point set Fix $(U)$. The mapping $U$ is said to be demiclosed at zero if for any $\left\{x_{n}\right\}$ in $H$, the following implication holds:

$$
x_{n} \rightarrow x \text { and }(I-U) x_{n} \rightarrow 0 \Longrightarrow x \in \operatorname{Fix}(U) .
$$

The mapping $U$ is called: 
- nonexpansive if $\|U x-U y\| \leq\|x-y\|$, for all $x, y \in H$.

- quasi-nonexpansive if $F i x(U) \neq \emptyset$ and

$$
\|U x-z\| \leq\|x-z\|, \quad \forall z \in \operatorname{Fix}(U), x \in H,
$$

or equivalently

$$
\langle U x-x, x-z\rangle \leq-\frac{1}{2}\|x-U x\|^{2}, \quad \forall z \in \operatorname{Fix}(U), x \in H,
$$

or equivalently

$$
\langle U x-z, x-z\rangle \leq\|x-z\|^{2}-\frac{1}{2}\|x-U x\|^{2}, \quad \forall z \in F i x(U), x \in H .
$$

(iii) $\beta$-demicontractive with $0 \leq \beta<1$ if $F i x(U) \neq \emptyset$ and

$$
\|U x-z\|^{2} \leq\|x-z\|^{2}+\beta\|(I-U) x\|^{2}, \quad \forall z \in F i x(U), x \in H .
$$

The class of nonexpansive mappings is contained properly in the one of quasinonexpansive mapping. The latter is a proper subclass of the class of demicontractive mappings. In this paper, we consider the class of quasi-nonexpansive mappings. Note that if $U$ is a $\beta$-demicontractive mapping then $U_{w}=(1-w) I+$ $w U$ with $w \in(0,1-\beta]$ is quasi-nonexpansive and Fix $\left(U_{w}\right)=F i x(U)$. Thus, if $U$ is $\beta$-demicontractive then the result in this paper is still true by replacing $U$ by $U_{w}$ for some $w \in(0,1-\beta]$. An example for the quasi-nonexpansive mapping is the subgradient projection. Let $g: H \rightarrow \Re$ be a convex function such that $\operatorname{lev}_{\leq g}:=\{x \in H: g(x) \leq 0\} \neq \emptyset$. The subgradient projection is a mapping defined by

$$
U x= \begin{cases}x-\frac{g(x)}{\|z(x)\|^{2}} z(x), & \text { if } g(x)>0 \\ x, & \text { otherwise }\end{cases}
$$

where $z(x) \in \partial g(x)$. In that case, $U$ is quasi-nonexpansive, demiclosed at zero, and $F i x(U)=\operatorname{lev}_{\leq g}$ (see, e.g., [3, Lemma 3.1]).

In any Hilbert space, we have the following results.

Lemma 1. Let $H$ be a real Hilbert space. Then the following results hold:

(i) $\|x+y\|^{2}=\|x\|^{2}+2\langle x, y\rangle+\|y\|^{2}, \forall x, y \in H$;

(ii) $\|x+y\|^{2} \leq\|x\|^{2}+2\langle y, x+y\rangle, \forall x, y \in H$.

The metric projection $P_{C}: H \rightarrow C$ is defined by, for each $x \in H, P_{C}(x) \in C$ such that $\left\|P_{C}(x)-x\right\| \leq\|y-x\| \forall y \in C$. It is well-known that the metric projection has the following properties.

Lemma 2. Let $P_{C}: H \rightarrow C$ be the metric projection from $H$ onto $C$. Then

(i) $\left\|x-P_{C} y\right\|^{2}+\left\|P_{C} y-y\right\|^{2} \leq\|x-y\|^{2}, \forall x \in C, y \in H$.

(ii) $z=P_{C} x$ if and only if $\langle x-z, z-y\rangle \geq 0, \quad \forall y \in C$. 
The proximal mapping of a proper, convex and lower semicontinuous function $g: C \rightarrow \Re$ with a parameter $\lambda>0$ is defined by

$$
\operatorname{prox}_{\lambda g}(x)=\arg \min \left\{\lambda g(y)+0.5\|x-y\|^{2}: y \in C\right\}, x \in H .
$$

The following is a property of the proximal mapping (see [4] for more details).

Lemma 3. For all $x \in H, y \in C$ and $\lambda>0$, the following inequality holds,

$$
\lambda\left\{g(y)-g\left(\operatorname{prox}_{\lambda g}(x)\right)\right\} \geq\left\langle x-\operatorname{prox}_{\lambda g}(x), y-\operatorname{prox}_{\lambda g}(x)\right\rangle .
$$

Remark 1. From Lemma 3, it is easy to show that if $x=\operatorname{prox}_{\lambda g}(x)$ then

$$
x \in \operatorname{Arg} \min \{g(y): y \in C\}:=\left\{x \in C: g(x)=\min _{y \in C} g(y)\right\}
$$

Lemma 4. ([24]) Let $\left\{a_{n}\right\}$ be a sequence of nonnegative real numbers such that there exists a subsequence $\left\{a_{n_{j}}\right\}$ of $\left\{a_{n}\right\}$ such that $a_{n_{j}}<a_{n_{j}+1}$ for all $j \geq 0$. Then there exists a nondecreasing sequence $\left\{m_{k}\right\}$ such that $\lim _{k \rightarrow \infty} m_{k}=\infty$ and the following properties are satisfied by all (sufficiently large) number $k \geq 0$ :

$$
a_{m_{k}} \leq a_{m_{k}+1} \text { and } a_{k} \leq a_{m_{k}+1}
$$

In fact, $m_{k}$ is the largest number $n$ in the set $\{1,2, \ldots, k\}$ such that $a_{n}<a_{n+1}$.

Lemma 5. ( $[33,38])$ Let $\left\{a_{n}\right\}$ be sequences of nonnegative real numbers such that:

$$
a_{n+1} \leq\left(1-\alpha_{n}\right) a_{n}+\alpha_{n} b_{n}
$$

where $\left\{\alpha_{n}\right\} \subset(0,1)$ and $\left\{b_{n}\right\}$ is a sequence such that (i) $\sum_{n=0}^{\infty} \alpha_{n}=\infty$ (ii) $\lim \sup _{n \rightarrow \infty} b_{n} \leq 0$. Then $\lim _{n \rightarrow \infty} a_{n}=0$.

\section{Algorithm and convergence analysis}

In this section, we present and analyze the strong convergence of a new algorithm for finding a common solution of an equilibrium problem for a bifunction $f: H \times H \rightarrow \Re$ and a fixed point problem for a quasi - nonexpansive mapping $U: H \rightarrow H$. In order to get the convergence of the algorithm, we consider the following assumptions:

- A1. $f$ is pseudomonotone on $C$ and $f(x, x)=0$ for all $x \in C$;

- A2. $f$ satisfies a Lipschitz-type condition on $H$ with the two constants $c_{1}, c_{2}$

- A3. $f(x,$.$) is convex and lower semicontinuos on C$ for every fixed $x \in H$;

- A4. $\lim \sup _{n \rightarrow \infty} f\left(x_{n}, y\right) \leq f(x, y)$ for each sequence $\left\{x_{n}\right\} \subset C$ converging weakly to $x$. 
It is easy to show that under assumptions $\mathrm{A} 1$ and $\mathrm{A} 3$, the solution set $\operatorname{EP}(f, C)$ is closed and convex. Since the set of fixed points of a quasi - nonexpansive mapping $U$ is closed and convex, the solution set $\Omega=E P(f, C) \cap F i x(U)$ is also closed and convex. Throughout this paper, the solution set $\Omega$ is assumed to be nonempty. Moreover, we consider the sequence of stepsizes $\left\{\lambda_{n}\right\} \subset(0,+\infty)$ and two parameter sequences $\left\{\alpha_{n}\right\} \subset[0,1]$ and $\left\{\beta_{n}\right\} \subset[0,1]$ satisfying the following conditions:

- B1. $0<\underline{\lambda} \leq \lambda_{n} \leq \bar{\lambda}<\min \left\{1 / 2 c_{1}, 1 / 2 c_{2}\right\}$.

- B2. $\lim _{n \rightarrow \infty} \alpha_{n}=0, \sum_{n=1}^{\infty} \alpha_{n}=+\infty$.

- B3. $\beta_{n} \subset(a, b) \subset(0,1)$ for some $b>a>0$.

Now, for approximating a solution in $\Omega=E P(f, C) \cap F i x(U)$, we investigate the asymptotic behavior of the sequence $\left\{x_{n}\right\}$ generated, from an arbitrary point $x_{0} \in H$, by the following algorithm:

Algorithm 1. (Mann proximal - like algorithm for EPs and FPPs)

Initialization:

Choose $x_{0} \in H$ and three sequences $\left\{\lambda_{n}\right\} \subset(0,+\infty),\left\{\alpha_{n}\right\} \subset[0,1]$, $\left\{\beta_{n}\right\} \subset[0,1]$ such that conditions B1 - B3 above hold.

\section{Iterative Steps:}

Assume that $x_{n} \in H$ is known, calculate $x_{n+1}$ as follows:

Step 1. Compute

$$
y_{n}=\operatorname{prox}_{\lambda_{n} f\left(x_{n}, .\right)}\left(x_{n}\right) \text { and } z_{n}=\operatorname{prox}_{\lambda_{n} f\left(y_{n}, .\right)}\left(x_{n}\right) .
$$

Step 2. Compute

$$
x_{n+1}=\left(1-\alpha_{n}-\beta_{n}\right) z_{n}+\beta_{n} U z_{n} .
$$

Set $n=: n+1$ and go back Step 1 .

It is well known that the hybrid methods with the normal Mann iteration only have weak convergence. However, Algorithm 1, which is incorporated with the similar method to the normal Mann iteration in Step 2, will be proved to be strongly convergent to an element in $\Omega$. More precisely, we have the following main result.

Theorem 1. Under assumptions A1-A4 and B1-B3, then the sequence $\left\{x_{n}\right\}$ generated by Algorithm 1 converges strongly to an element in $\Omega=E P(f, C) \cap$ $\operatorname{Fix}(U)$.

Before presenting the proof of Theorem 1, we need the following lemmas. We begin with an important result which will be used to prove the next two lemmas.

Lemma 6. For all $y \in C$, we have the following estimate,

$$
\begin{gathered}
2 \lambda_{n} f\left(y_{n}, y\right) \geq\left(1-2 c_{1} \lambda_{n}\right)\left\|x_{n}-y_{n}\right\|^{2}+\left(1-2 c_{2} \lambda_{n}\right)\left\|z_{n}-y_{n}\right\|^{2} \\
+\left\|z_{n}-y\right\|^{2}-\left\|x_{n}-y\right\|^{2} .
\end{gathered}
$$


Proof. It follows from Lemma 3 and the definition of $z_{n}$ that

$$
\lambda_{n}\left\{f\left(y_{n}, y\right)-f\left(y_{n}, z_{n}\right)\right\} \geq\left\langle z_{n}-x_{n}, z_{n}-y\right\rangle, \forall y \in C .
$$

Thus, multiplying both two sides of this inequality by 2 and using the equality $2\langle a, b\rangle=\|a\|^{2}+\|b\|^{2}-\|a-b\|^{2}$, we obtain

$$
\begin{aligned}
2 \lambda_{n} f\left(y_{n}, y\right) & \geq 2 \lambda_{n} f\left(y_{n}, z_{n}\right)+2\left\langle z_{n}-x_{n}, z_{n}-y\right\rangle \\
& =2 \lambda_{n} f\left(y_{n}, z_{n}\right)+\left\|z_{n}-x_{n}\right\|^{2}+\left\|z_{n}-y\right\|^{2}-\left\|x_{n}-y\right\|^{2} .
\end{aligned}
$$

Similarly, from Lemma 3 and the definition of $y_{n}$, we obtain

$$
\lambda_{n}\left(f\left(x_{n}, y\right)-f\left(x_{n}, y_{n}\right)\right) \geq\left\langle y_{n}-x_{n}, y_{n}-y\right\rangle, \forall y \in C,
$$

which, with $y=z_{n} \in C$, implies that

$$
\lambda_{n}\left(f\left(x_{n}, z_{n}\right)-f\left(x_{n}, y_{n}\right)\right) \geq\left\langle y_{n}-x_{n}, y_{n}-z_{n}\right\rangle .
$$

This together with the Lipschitz-type condition of $f$ and the equality $2\langle a, b\rangle=$ $\|a\|^{2}+\|b\|^{2}-\|a-b\|^{2}$ implies that

$$
\begin{array}{rl}
2 \lambda_{n} & f\left(y_{n}, z_{n}\right) \geq 2 \lambda_{n}\left\{f\left(x_{n}, z_{n}\right)-f\left(x_{n}, y_{n}\right)-c_{1}\left\|x_{n}-y_{n}\right\|^{2}-c_{2}\left\|z_{n}-y_{n}\right\|^{2}\right\} \\
& =2 \lambda_{n}\left\{f\left(x_{n}, z_{n}\right)-f\left(x_{n}, y_{n}\right)\right\}-2 c_{1} \lambda_{n}\left\|x_{n}-y_{n}\right\|^{2}-2 c_{2} \lambda_{n}\left\|z_{n}-y_{n}\right\|^{2} \\
& \geq 2\left\langle y_{n}-x_{n}, y_{n}-z_{n}\right\rangle-2 c_{1} \lambda_{n}\left\|x_{n}-y_{n}\right\|^{2}-2 c_{2} \lambda_{n}\left\|z_{n}-y_{n}\right\|^{2} \\
& =\left(1-2 c_{1} \lambda_{n}\right)\left\|x_{n}-y_{n}\right\|^{2}+\left(1-2 c_{2} \lambda_{n}\right)\left\|z_{n}-y_{n}\right\|^{2}-\left\|x_{n}-z_{n}\right\|^{2} .
\end{array}
$$

From relations (3.1) and (3.2), we obtain the desired conclusion. Lemma 6 is proved.

Using Lemma 6, we will prove the boundedness of the sequences generated by Algorithm 1.

Lemma 7. The sequences $\left\{x_{n}\right\},\left\{y_{n}\right\},\left\{z_{n}\right\}$ are bounded.

Proof. Let $p \in \Omega=E P(f, C) \cap F i x(U)$. It follows from the definiton of $x_{n+1}$ and the triangle inequality that

$$
\begin{aligned}
\left\|x_{n+1}-p\right\| & =\left\|\left(1-\alpha_{n}-\beta_{n}\right)\left(z_{n}-p\right)+\beta_{n}\left(U z_{n}-p\right)-\alpha_{n} p\right\| \\
& \leq\left\|\left(1-\alpha_{n}-\beta_{n}\right)\left(z_{n}-p\right)+\beta_{n}\left(U z_{n}-p\right)\right\|+\alpha_{n}\|p\| .
\end{aligned}
$$

Since $\alpha_{n} \rightarrow 0$ and $0<a \leq \beta_{n} \leq b<1$, there exists a number $n_{0} \geq 0$ such that $1-\alpha_{n}-\beta_{n}>0$, and so $\beta_{n}\left(1-\alpha_{n}-\beta_{n}\right)>0$ for all $n \geq n_{0}$. Without loss of generality, throughout this section, we can assume that $\beta_{n}\left(1-\alpha_{n}-\beta_{n}\right)>0$ for all $n \geq 0$. Thus, from the quasi-nonexpansiveness of $U$ and the equality $\left(1-\alpha_{n}-\beta_{n}\right)^{2}+2\left(1-\alpha_{n}-\beta_{n}\right) \beta_{n}+\beta_{n}^{2}=\left(1-\alpha_{n}\right)^{2}$, we obtain the following estimate,

$$
\begin{gathered}
\left\|\left(1-\alpha_{n}-\beta_{n}\right)\left(z_{n}-p\right)+\beta_{n}\left(U z_{n}-p\right)\right\|^{2}=\left(1-\alpha_{n}-\beta_{n}\right)^{2}\left\|z_{n}-p\right\|^{2} \\
+2\left(1-\alpha_{n}-\beta_{n}\right) \beta_{n}\left\langle U z_{n}-p, z_{n}-p\right\rangle+\beta_{n}^{2}\left\|U z_{n}-p\right\|^{2}
\end{gathered}
$$




$$
\begin{aligned}
= & \left(1-\alpha_{n}-\beta_{n}\right)^{2}\left\|z_{n}-p\right\|^{2}+\left(1-\alpha_{n}-\beta_{n}\right) \beta_{n}\left(\left\|U z_{n}-p\right\|^{2}\right. \\
& \left.+\left\|z_{n}-p\right\|^{2}-\left\|z_{n}-U z_{n}\right\|^{2}\right)+\beta_{n}^{2}\left\|U z_{n}-p\right\|^{2} \\
\leq & \left(1-\alpha_{n}-\beta_{n}\right)^{2}\left\|z_{n}-p\right\|^{2}+\left(1-\alpha_{n}-\beta_{n}\right) \beta_{n}\left(2\left\|z_{n}-p\right\|^{2}-\left\|z_{n}-U z_{n}\right\|^{2}\right) \\
& +\beta_{n}^{2}\left\|z_{n}-p\right\|^{2}=\left(1-\alpha_{n}\right)^{2}\left\|z_{n}-p\right\|^{2}-\beta_{n}\left(1-\alpha_{n}-\beta_{n}\right)\left\|z_{n}-U z_{n}\right\|^{2} \\
\leq & \left(1-\alpha_{n}\right)^{2}\left\|z_{n}-p\right\|^{2} .
\end{aligned}
$$

This implies that

$$
\left\|\left(1-\alpha_{n}-\beta_{n}\right)\left(z_{n}-p\right)+\beta_{n}\left(U x_{n}-p\right)\right\| \leq\left(1-\alpha_{n}\right)\left\|z_{n}-p\right\|,
$$

which together with relation (3.3), implies that

$$
\left\|x_{n+1}-p\right\| \leq\left(1-\alpha_{n}\right)\left\|z_{n}-p\right\|+\alpha_{n}\|p\| .
$$

Since $p \in E P(f, C)$ and $y_{n} \in C, f\left(p, y_{n}\right) \geq 0$ for all $n \geq 0$. This together with the pseudomonotonicity of $f$ implies that $f\left(y_{n}, p\right) \leq 0$. Thus, using Lemma 6 with $y=p \in C$, we come to the following estimate

$$
\left\|z_{n}-p\right\|^{2} \leq\left\|x_{n}-p\right\|^{2}-\left(1-2 c_{1} \lambda_{n}\right)\left\|x_{n}-y_{n}\right\|^{2}-\left(1-2 c_{2} \lambda_{n}\right)\left\|z_{n}-y_{n}\right\|^{2},
$$

which, together with hypothesis B1, implies that $\left\|z_{n}-p\right\| \leq\left\|x_{n}-p\right\|$. Thus, from (3.4), we get

$$
\begin{aligned}
& \left\|x_{n+1}-p\right\| \leq\left(1-\alpha_{n}\right)\left\|x_{n}-p\right\|+\alpha_{n}\|p\| \\
& \quad \leq \max \left\{\left\|x_{n}-p\right\|,\|p\|\right\} \leq \ldots \leq \max \left\{\left\|x_{0}-p\right\|,\|p\|\right\} .
\end{aligned}
$$

This says that the sequence $\left\{x_{n}\right\}$ is bounded, and so $\left\{z_{n}\right\}$ is. Furthermore, the boundedness of $\left\{y_{n}\right\}$ follows from (3.5). This completes the proof of Lemma 7 .

Next, we are interested in the weak cluster points of the sequence $\left\{x_{n}\right\}$. We have the following result.

Lemma 8. If $\left\{x_{n_{k}}\right\}$ is a subsequence of $\left\{x_{n}\right\}$ such that $x_{n_{k}} \rightarrow p,\left\|x_{n_{k}}-y_{n_{k}}\right\| \rightarrow$ $0,\left\|y_{n_{k}}-z_{n_{k}}\right\| \rightarrow 0$ and $\left\|z_{n_{k}}-U z_{n_{k}}\right\| \rightarrow 0$, then $p \in \Omega=E P(f, C) \cap F i x(U)$.

Proof. It follows from the hypotheses of Lemma 8 that $y_{n_{k}} \rightarrow p$ and $z_{n_{k}} \rightarrow p$ as $k \rightarrow \infty$. Moreover,

$$
\begin{aligned}
\left\|\mid z_{n_{k}}-y\right\|^{2} & -\left\|x_{n_{k}}-y\right\|^{2}|=|\left\|z_{n_{k}}-y\right\|-\left\|x_{n_{k}}-y\right\| \|\left(\left\|z_{n_{k}}-y\right\|+\left\|x_{n_{k}}-y\right\|\right) \\
& \leq\left\|z_{n_{k}}-x_{n_{k}}\right\|\left(\left\|z_{n_{k}}-y\right\|+\left\|x_{n_{k}}-y\right\|\right) \\
& \leq\left(\left\|z_{n_{k}}-y_{n_{k}}\right\|+\left\|x_{n_{k}}-y_{n_{k}}\right\|\right)\left(\left\|z_{n_{k}}-y\right\|+\left\|x_{n_{k}}-y\right\|\right) .
\end{aligned}
$$

Thus, also from the hypotheses of Lemma 8 and the boundedness of $\left\{x_{n_{k}}\right\}$ and $\left\{z_{n_{k}}\right\}$, we obtain

$$
\left\|z_{n_{k}}-y\right\|^{2}-\left\|x_{n_{k}}-y\right\|^{2} \rightarrow 0 .
$$


From Lemma 6 with noting that $\lambda_{n_{k}} \geq \underline{\lambda}>0$, we obtain for all $y \in C$ that

$$
\begin{aligned}
f\left(y_{n_{k}}, y\right) \geq & \frac{1-2 c_{1} \lambda_{n_{k}}}{2 \lambda_{n_{k}}}\left\|x_{n_{k}}-y_{n_{k}}\right\|^{2}+\frac{1-2 c_{2} \lambda_{n_{k}}}{2 \lambda_{n_{k}}}\left\|z_{n_{k}}-y_{n_{k}}\right\|^{2} \\
& +\frac{\left\|z_{n_{k}}-y\right\|^{2}-\left\|x_{n_{k}}-y\right\|^{2}}{2 \lambda_{n_{k}}} .
\end{aligned}
$$

Passing to the limit in the last inequality, using relation (3.6), the hypotheses of Lemma 8 and A4, we obtain $f(p, y) \geq 0, \forall y \in C$. Thus, $p \in E P(f, C)$. Moreover, from $\left\|z_{n_{k}}-U z_{n_{k}}\right\| \rightarrow 0$, the demi-closedness at zero of $U$ and $z_{n_{k}} \rightarrow p$, we get that $p \in F i x(U)$. Then, $p \in E P(f, C) \cap F i x(U)$. This finishes the proof of Lemma 8 .

Since $\left\{z_{n}\right\}$ and $\left\{x_{n}\right\}$ are bounded, there exists $M_{p}>0$ for each $p \in \Omega$ such that

$$
2 \sup _{n \geq 0}\left|\left\langle z_{n}, p-x_{n+1}\right\rangle\right| \leq M_{p}
$$

We have the following lemma.

Lemma 9. The following estimates hold for all $p \in \Omega$,

(i) $\beta_{n}\left(1-\beta_{n}\right)\left\|z_{n}-U z_{n}\right\|^{2} \leq\left\|x_{n}-p\right\|^{2}-\left\|x_{n+1}-p\right\|^{2}+\alpha_{n} M_{p}$,

(ii) $\left\|x_{n+1}-p\right\|^{2} \leq\left(1-\alpha_{n}\right)\left\|x_{n}-p\right\|^{2}+2 \alpha_{n} \beta_{n}\left\|z_{n}-U z_{n}\right\|\left\|x_{n+1}-p\right\|$ $+2 \alpha_{n}\left\langle p, p-x_{n+1}\right\rangle$.

Proof. (i). It follows from the definiton of $x_{n+1}$ and Lemma 1 that

$$
\begin{aligned}
\left\|x_{n+1}-p\right\|^{2}= & \left\|\left(z_{n}-p\right)+\beta_{n}\left(U z_{n}-z_{n}\right)-\alpha_{n} z_{n}\right\|^{2} \\
\leq & \left\|\left(z_{n}-p\right)+\beta_{n}\left(U z_{n}-z_{n}\right)\right\|^{2}-2 \alpha_{n}\left\langle z_{n}, x_{n+1}-p\right\rangle \\
= & \left\|z_{n}-p\right\|^{2}+\beta_{n}^{2}\left\|U z_{n}-z_{n}\right\|^{2}+2 \beta_{n}\left\langle U z_{n}-z_{n}, z_{n}-p\right\rangle \\
& +2 \alpha_{n}\left\langle z_{n}, p-x_{n+1}\right\rangle .
\end{aligned}
$$

On the other hand, from Lemma 1(i) and the quasi-nonexpansiveness of $U$, we have that

$2\left\langle U z_{n}-z_{n}, z_{n}-p\right\rangle=\left\|U z_{n}-p\right\|^{2}-\left\|z_{n}-p\right\|^{2}-\left\|U z_{n}-z_{n}\right\|^{2} \leq-\left\|U z_{n}-z_{n}\right\|^{2}$,

which, together with relations (3.7), (3.8) and the fact $\left\|z_{n}-p\right\| \leq\left\|x_{n}-p\right\|$, implies that

$$
\begin{aligned}
\left\|x_{n+1}-p\right\|^{2} & \leq\left\|z_{n}-p\right\|^{2}+\beta_{n}^{2}\left\|U z_{n}-z_{n}\right\|^{2}-\beta_{n}\left\|z_{n}-U z_{n}\right\|^{2}+2 \alpha_{n}\left\langle z_{n}, p-x_{n+1}\right\rangle \\
& \leq\left\|z_{n}-p\right\|^{2}-\beta_{n}\left(1-\beta_{n}\right)\left\|z_{n}-U z_{n}\right\|^{2}+\alpha_{n} M_{p} \\
& \leq\left\|x_{n}-p\right\|^{2}-\beta_{n}\left(1-\beta_{n}\right)\left\|z_{n}-U z_{n}\right\|^{2}+\alpha_{n} M_{p}
\end{aligned}
$$

This leads to conclusion (i).

(ii). Setting $t_{n}=\left(1-\beta_{n}\right) z_{n}+\beta_{n} U z_{n}$, and so from the definition of $x_{n+1}$ we 
obtain that $x_{n+1}=t_{n}-\alpha_{n} z_{n}$. It follows from Lemma 1(i) and the quasinonexpansiveness of $U$ that

$$
\begin{aligned}
\| t_{n}- & p\left\|^{2}=\right\|\left(1-\beta_{n}\right)\left(z_{n}-p\right)+\beta_{n}\left(U z_{n}-p\right) \|^{2} \\
= & \left(1-\beta_{n}\right)^{2}\left\|z_{n}-p\right\|^{2}+\beta_{n}^{2}\left\|U z_{n}-p\right\|^{2}+2\left(1-\beta_{n}\right) \beta_{n}\left\langle U z_{n}-p, z_{n}-p\right\rangle \\
= & \left(1-\beta_{n}\right)^{2}\left\|z_{n}-p\right\|^{2}+\beta_{n}^{2}\left\|U z_{n}-p\right\|^{2} \\
& +\left(1-\beta_{n}\right) \beta_{n}\left\{\left\|U z_{n}-p\right\|^{2}+\left\|z_{n}-p\right\|^{2}-\left\|U z_{n}-z_{n}\right\|^{2}\right\} \\
\leq & \left(1-\beta_{n}\right)^{2}\left\|z_{n}-p\right\|^{2}+\beta_{n}^{2}\left\|z_{n}-p\right\|^{2} \\
& +\left(1-\beta_{n}\right) \beta_{n}\left\{2\left\|z_{n}-p\right\|^{2}-\left\|U z_{n}-z_{n}\right\|^{2}\right\} \\
= & \left\|z_{n}-p\right\|^{2}-\beta_{n}\left(1-\beta_{n}\right)\left\|U z_{n}-z_{n}\right\|^{2},
\end{aligned}
$$

in which the last equality follows from the fact $\left(1-\beta_{n}\right)^{2}+\beta_{n}^{2}+2\left(1-\beta_{n}\right) \beta_{n}=1$. By condition B3, relation (3.11) and the fact $\left\|z_{n}-p\right\| \leq\left\|x_{n}-p\right\|$, we get $\left\|t_{n}-p\right\|^{2} \leq\left\|z_{n}-p\right\|^{2} \leq\left\|x_{n}-p\right\|^{2}$. Thus,

$$
\left\|t_{n}-p\right\| \leq\left\|x_{n}-p\right\| .
$$

From the definitions of $x_{n+1}$ and $t_{n}$, we obtain

$$
\begin{aligned}
x_{n+1} & =t_{n}-\alpha_{n} z_{n}=\left(1-\alpha_{n}\right) t_{n}-\alpha_{n}\left(z_{n}-t_{n}\right) \\
& =\left(1-\alpha_{n}\right) t_{n}-\alpha_{n} \beta_{n}\left(z_{n}-U z_{n}\right),
\end{aligned}
$$

which, together with Lemma 1(ii), the inequality $\langle a, b\rangle \leq\|a\| \||b| \mid$ and relation (3.12), implies that

$$
\begin{aligned}
& \left\|x_{n+1}-p\right\|^{2}=\left\|\left(1-\alpha_{n}\right)\left(t_{n}-p\right)-\left(\alpha_{n} \beta_{n}\left(z_{n}-U z_{n}\right)+\alpha_{n} p\right)\right\|^{2} \\
& \quad \leq\left(1-\alpha_{n}\right)^{2}\left\|t_{n}-p\right\|^{2}-2\left\langle\alpha_{n} \beta_{n}\left(z_{n}-U z_{n}\right)+\alpha_{n} p, x_{n+1}-p\right\rangle \\
& \quad=\left(1-\alpha_{n}\right)^{2}\left\|t_{n}-p\right\|^{2}+2 \alpha_{n} \beta_{n}\left\langle z_{n}-U z_{n}, p-x_{n+1}\right\rangle+2 \alpha_{n}\left\langle p, p-x_{n+1}\right\rangle \\
& \leq\left(1-\alpha_{n}\right)\left\|t_{n}-p\right\|^{2}+\alpha_{n}\left(2 \beta_{n}\left\|z_{n}-U z_{n}\right\|\left\|x_{n+1}-p\right\|+2\left\langle p, p-x_{n+1}\right\rangle\right) \\
& \quad \leq\left(1-\alpha_{n}\right)\left\|x_{n}-p\right\|^{2}+\alpha_{n}\left(2 \beta_{n}\left\|z_{n}-U z_{n}\right\|\left\|x_{n+1}-p\right\|+2\left\langle p, p-x_{n+1}\right\rangle\right) .
\end{aligned}
$$

This completes the proof of Lemma 9.

The proof of Theorem 1.

Proof. Since $\Omega$ is nonempty closed and convex set, there exists a unique element $p^{\dagger} \in \Omega$ such that $p^{\dagger}=P_{\Omega}(0)$. This, from Lemma 2(ii), is equivalent to the following inequality,

$$
\left\langle p^{\dagger}, p^{\dagger}-p\right\rangle \leq 0, \quad \forall p \in \Omega
$$

We will prove the sequence $\left\{\left\|x_{n}-p^{\dagger}\right\|^{2}\right\}$ converges to zero as $n \rightarrow \infty$. Indeed, using Lemma 9 with $p=p^{\dagger} \in \Omega$ and setting $M=M_{p^{\dagger}}>0$, we can rewrite those inequalities as follows:

$$
\beta_{n}\left(1-\beta_{n}\right)\left\|z_{n}-U z_{n}\right\|^{2} \leq\left\|x_{n}-p^{\dagger}\right\|^{2}-\left\|x_{n+1}-p^{\dagger}\right\|^{2}+\alpha_{n} M .
$$




$$
\left\|x_{n+1}-p^{\dagger}\right\|^{2} \leq\left(1-\alpha_{n}\right)\left\|x_{n}-p^{\dagger}\right\|^{2}+\alpha_{n} b_{n}
$$

where $b_{n}=2 \beta_{n}\left\|z_{n}-U z_{n}\right\|\left\|x_{n+1}-p^{\dagger}\right\|+2\left\langle p^{\dagger}, p^{\dagger}-x_{n+1}\right\rangle$. Now, we consider two possible cases on the sequence $\left\{\left\|x_{n}-p^{\dagger}\right\|^{2}\right\}$.

Case 1: There exists an integer number $n_{0} \geq 0$ such that $\left\|x_{n+1}-p^{\dagger}\right\|^{2} \leq$ $\left\|x_{n}-p^{\dagger}\right\|^{2}$ for all $n \geq n_{0}$. This means that the sequence $\left\{\left\|x_{n}-p^{\dagger}\right\|^{2}\right\}_{n \geq n_{0}}$ is non-increasing. Thus, the limit of $\left\|x_{n}-p^{\dagger}\right\|^{2}$ exists. This together with relation (3.14), hypotheses B2 and B3 implies that

$$
\lim _{n \rightarrow \infty}\left\|z_{n}-U z_{n}\right\|=0
$$

Moreover, recalling relations (3.9) and (3.10) with $p=p^{\dagger}$ and $M=M_{p^{\dagger}}>0$, we have

$$
\begin{aligned}
\left\|x_{n+1}-p^{\dagger}\right\|^{2} & \leq\left\|z_{n}-p^{\dagger}\right\|^{2}-\beta_{n}\left(1-\beta_{n}\right)\left\|z_{n}-U z_{n}\right\|^{2}+\alpha_{n} M \\
& \leq\left\|x_{n}-p^{\dagger}\right\|^{2}-\beta_{n}\left(1-\beta_{n}\right)\left\|z_{n}-U z_{n}\right\|^{2}+\alpha_{n} M
\end{aligned}
$$

Passing to the limit in the last inequality and using relation (3.16), $\alpha_{n} \rightarrow 0$, we obtain that $\lim _{n \rightarrow \infty}\left\|z_{n}-p^{\dagger}\right\|^{2}=\lim _{n \rightarrow \infty}\left\|x_{n}-p^{\dagger}\right\|^{2}$. Hence, $\left\|z_{n}-p^{\dagger}\right\|^{2}-$ $\left\|x_{n}-p^{\dagger}\right\|^{2} \rightarrow 0$ which, together with relation (3.5) and hypothesis B1, implies that

$$
\left\|y_{n}-x_{n}\right\| \rightarrow 0 \text { and }\left\|z_{n}-y_{n}\right\| \rightarrow 0 .
$$

Thus, from the triangle inequality, we obtain that $\left\|z_{n}-x_{n}\right\| \rightarrow 0$. Hence, from the definition of $x_{n+1}$, the triangle inequality and relation (3.16), we obtain that

$$
\left\|x_{n+1}-x_{n}\right\| \leq\left\|z_{n}-x_{n}\right\|+\alpha_{n}\left\|z_{n}\right\|+\beta_{n}\left\|z_{n}-U z_{n}\right\| \rightarrow 0 \text { as } n \rightarrow \infty .
$$

Since $\left\{x_{n}\right\}$ is bounded, without loss generality, we can assume that there exists a subsequence $\left\{x_{n_{j}}\right\}$ of $\left\{x_{n}\right\}$ such that $x_{n_{j}} \rightarrow p$ and

$$
\limsup _{n \rightarrow \infty}\left\langle p^{\dagger}, p^{\dagger}-x_{n}\right\rangle=\lim _{j \rightarrow \infty}\left\langle p^{\dagger}, p^{\dagger}-x_{n_{j}}\right\rangle=\left\langle p^{\dagger}, p^{\dagger}-p\right\rangle .
$$

Thus, from relations (3.16), (3.17) and Lemma 8, we obtain that $p \in \Omega$. This together with $\left\|x_{n+1}-x_{n}\right\| \rightarrow 0$ and relations (3.13), (3.19) implies that $\lim \sup _{n \rightarrow \infty}\left\langle p^{\dagger}, p^{\dagger}-x_{n+1}\right\rangle \leq 0$. Hence, it follows from the definiton of $b_{n}$ and relation (3.16) that $\limsup _{n \rightarrow \infty} b_{n} \leq 0$. Combining this with relation (3.15), hypothesis B2 and Lemma 5 , we obtain that $\lim _{n \rightarrow \infty}\left\|x_{n}-p^{\dagger}\right\|^{2}=0$, i.e., $x_{n} \rightarrow p^{\dagger}$ as $n \rightarrow \infty$.

Case 2: There exists a subsequence $\left\{\left\|x_{n_{j}}-p^{\dagger}\right\|^{2}\right\}$ of $\left\{\left\|x_{n}-p^{\dagger}\right\|^{2}\right\}$ such that $\left\|x_{n_{j}}-p^{\dagger}\right\|^{2}<\left\|x_{n_{j}+1}-p^{\dagger}\right\|^{2}$ for all $j \geq 0$. In this case, it follows from Lemma 4 that there exists a non-decreasing sequence $\left\{m_{k}\right\}$ such that $\lim _{k \rightarrow \infty} m_{k}=\infty$ and the following inequalities hold for all $k \geq 0$ :

$$
\left\|x_{m_{k}}-p^{\dagger}\right\|^{2} \leq\left\|x_{m_{k}+1}-p^{\dagger}\right\|^{2} \text { and }\left\|x_{k}-p^{\dagger}\right\|^{2} \leq\left\|x_{m_{k}+1}-p^{\dagger}\right\|^{2} .
$$


Thus, from relation (3.14), we obtain

$$
\beta_{m_{k}}\left(1-\beta_{m_{k}}\right)\left\|z_{m_{k}}-U z_{m_{k}}\right\|^{2} \leq\left\|x_{m_{k}}-p^{\dagger}\right\|^{2}-\left\|x_{m_{k}+1}-p^{\dagger}\right\|^{2}+\alpha_{m_{k}} M \leq \alpha_{m_{k}} M,
$$

which, together with hypotheses B2, B3, implies that

$$
\lim _{k \rightarrow \infty}\left\|z_{m_{k}}-U z_{m_{k}}\right\|=0
$$

On the other hand, it follows from relations (3.20) and (3.9), (3.10) with $p=p^{\dagger}$ and $M:=M_{p^{\dagger}}>0$ that

$$
\begin{aligned}
\left\|x_{m_{k}}-p^{\dagger}\right\|^{2} & \leq\left\|x_{m_{k}+1}-p^{\dagger}\right\|^{2} \\
& \leq\left\|z_{m_{k}}-p^{\dagger}\right\|^{2}-\beta_{m_{k}}\left(1-\beta_{m_{k}}\right)\left\|z_{m_{k}}-U z_{m_{k}}\right\|^{2}+\alpha_{m_{k}} M \\
& \left.\leq\left\|x_{m_{k}}-p^{\dagger}\right\|^{2}-\beta_{m_{k}}\left(1-\beta_{m_{k}}\right)\right]\left\|z_{m_{k}}-U z_{m_{k}}\right\|^{2}+\alpha_{m_{k}} M
\end{aligned}
$$

Thus, from relation (3.21) and B2, we obtain that $\left\|z_{m_{k}}-p^{\dagger}\right\|^{2}-\left\|x_{m_{k}}-p^{\dagger}\right\|^{2} \rightarrow 0$. By arguing similarly to relations (3.17) and (3.18) in Case 1, we obtain

$$
\begin{aligned}
& \left\|y_{m_{k}}-x_{m_{k}}\right\| \rightarrow 0, \quad\left\|z_{m_{k}}-y_{m_{k}}\right\| \rightarrow 0, \quad\left\|x_{m_{k}+1}-x_{m_{k}}\right\| \rightarrow 0, \\
& \underset{k \rightarrow \infty}{\limsup }\left\langle p^{\dagger}, p^{\dagger}-x_{m_{k}+1}\right\rangle \leq 0 .
\end{aligned}
$$

It follows from relations $(3.15),(3.20)$ and the definition of $b_{n}$ that

$$
\begin{aligned}
& \left\|x_{m_{k}+1}-p^{\dagger}\right\|^{2} \leq\left(1-\alpha_{m_{k}}\right)\left\|x_{m_{k}}-p^{\dagger}\right\|^{2} \\
& \quad+\alpha_{m_{k}}\left[2 \beta_{m_{k}}\left\|z_{m_{k}}-U z_{m_{k}}\right\|\left\|x_{m_{k}+1}-p^{\dagger}\right\|+2\left\langle p^{\dagger}, p^{\dagger}-x_{m_{k}+1}\right\rangle\right] \\
& \leq\left(1-\alpha_{m_{k}}\right)\left\|x_{m_{k}+1}-p^{\dagger}\right\|^{2} \\
& \quad+\alpha_{m_{k}}\left[2 \beta_{m_{k}}\left\|z_{m_{k}}-U z_{m_{k}}\right\|\left\|x_{m_{k}+1}-p^{\dagger}\right\|+2\left\langle p^{\dagger}, p^{\dagger}-x_{m_{k}+1}\right\rangle\right] .
\end{aligned}
$$

A straightforward computation into the last inequality with noting that $\alpha_{m_{k}}>$ 0 implies that

$$
\left\|x_{m_{k}+1}-p^{\dagger}\right\|^{2} \leq 2 \beta_{m_{k}}\left\|z_{m_{k}}-U z_{m_{k}}\right\|\left\|x_{m_{k}+1}-p^{\dagger}\right\|+2\left\langle p^{\dagger}, p^{\dagger}-x_{m_{k}+1}\right\rangle .
$$

Combining this with relation (3.20), we get

$$
\left\|x_{k}-p^{\dagger}\right\|^{2} \leq 2 \beta_{m_{k}}\left\|z_{m_{k}}-U z_{m_{k}}\right\|\left\|x_{m_{k}+1}-p^{\dagger}\right\|+2\left\langle p^{\dagger}, p^{\dagger}-x_{m_{k}+1}\right\rangle,
$$

which, from relations (3.21) and (3.22), implies that lim $\sup _{k \rightarrow \infty}\left\|x_{k}-p^{\dagger}\right\|^{2} \leq 0$, i.e., $x_{k} \rightarrow p^{\dagger}$ as $k \rightarrow \infty$. This completes the proof of Theorem 1 .

\section{Computational experiments}

In this section, we consider three examples, one in a finite dimensional space and the two other ones in infinite dimensional spaces, to illustrate the convergence of the proposed algorithm (Algorithm 1) with the aim to compare its numerical behavior with four other strongly convergent algorithms, namely the extragradient-viscosity method (EGVM) introduced in [37, Algorithm 1], the 
hybrid extragradient method (HEGM) proposed in [36, Algorithm 1] and the shrinking extragradient method (SEGM) presented in [31, Theorem 4.2].

We use the sequence $D_{n}=\left\|x_{n}-x^{*}\right\|^{2}, n=0,1,2, \ldots$ to study the convergence of all the algorithms, where $x^{*}$ is the solution of the considered problem and $\left\{x_{n}\right\}$ is the sequence generated by each algorithm. The convergence of $D_{n}$ to zero implies that the sequence $\left\{x_{n}\right\}$ converges to the solution $x^{*}$ of the problem. All the programs are written in Malab 7.0 and computed on a PC Desktop Intel(R) Core(TM) i5-3210M CPU @ 2.50GHz RAM 2.00 G.B

Example 1. Consider our problem in the space $H=\Re^{m}$ for the equilibrium bifunction $f(x, y)=\langle P x+Q y+q, y-x\rangle$ and the mapping $U x$ is defined by the form (2.3) in Section 2, where $g: \Re^{m} \rightarrow \Re$ is a convex function such that $\operatorname{lev}_{\leq g}:=\left\{x \in \Re^{m}: g(x) \leq 0\right\} \neq \emptyset, z(x) \in \partial g(x), q \in \Re^{m}$ and $P, Q$ are two matrices of order $m$ such that $Q$ is symmetric positive semidefinite and $Q-P$ is symmetric negative semidefinite. The feasible set $C$ is given by

$$
C=\left\{x \in \Re^{m}: x_{i} \geq-1, \forall i=1, \ldots, m\right\} .
$$

In that case, $U$ is quasi-nonexpansive and demiclosed at zero, see, e.g., $[3$, Lemma 3.1] and Fix $(U)=\operatorname{lev}_{\leq g}$. Moreover, $f$ satisfies conditions A1-A4 with $c_{1}=c_{2}=\|P-Q\| / 2$. We choose the stepsize $\lambda_{n}=\frac{1}{2.1 c_{1}}$, the parameter sequence $\alpha_{n}=\frac{1}{\sqrt{n+1}}$ or $\alpha_{n}=\frac{1}{n+1}$, and $\beta_{n}=0.5\left(1-\alpha_{n}\right)$ for all the algorithms. The starting point in this example is $x_{0}=(1,1, \ldots, 1)^{T} \in \Re^{m}$.

For experiments, we choose $g(x)=\max \{0,\langle c, x\rangle+d\}$, where $d \in \Re_{-}$is a negative real number generated randomly and uniformly in $(-2,0), c$ is a vector in $\Re^{m}$ with its entries being generated randomly and uniformly in $(0,2)$ and $\bar{c} \in \partial g(x)$ (if $\langle c, x\rangle=-d$ ) has been chosen randomly. The vector $q$ equals to the zero and the two matrices $P, Q$ are generated randomly ${ }^{1}$ such that their conditions are satisfied. In that case the solution of the problem is $x^{*}=0$. All the optimization problems in the algorithms are effectively solved by Matlab Optimization Toolbox. Figures 1 and 2 describe the numerical results in $\Re^{100}$ while Figures 3 and 4 are in $\Re^{200}$.

Example 2. Consider our problem in the space $H=L^{2}[0,1]$, the feasible set $C$ is the unit ball $\mathrm{B}[0,1]$, the equilibrium bifunction $f$ is of the form $f(x, y)=$ $\langle A x, y-x\rangle$ with the operator $A: H \rightarrow H$ defined by

$$
A(x)(t)=\int_{0}^{1}\left[x(t)-F_{1}(t, s) f_{1}(x(s))\right] d s+g_{1}(t), x \in H, t \in[0,1],
$$

and the mapping $U: H \rightarrow H$ is given by

$$
U(x)(t)=\int_{0}^{1} F_{2}(t, s) f_{2}(x(s)) d s+g_{2}(t), x \in H, t \in[0,1] .
$$

\footnotetext{
${ }^{1}$ We randomly choose $\lambda_{1 k} \in(-2,0), \lambda_{2 k} \in(0,2), k=1, \ldots, m$. We set $\widehat{Q}_{1}, \widehat{Q}_{2}$ as two diagonal matrices with eigenvalues $\left\{\lambda_{1 k}\right\}_{k=1}^{m}$ and $\left\{\lambda_{2 k}\right\}_{k=1}^{m}$, respectively. Then, we construct a positive semidefinite matrix $Q$ and a negative semidefinite matrix $T$ by using random orthogonal matrices with $\widehat{Q}_{2}$ and $\widehat{Q}_{1}$, respectively. Finally, we set $P=Q-T$
} 


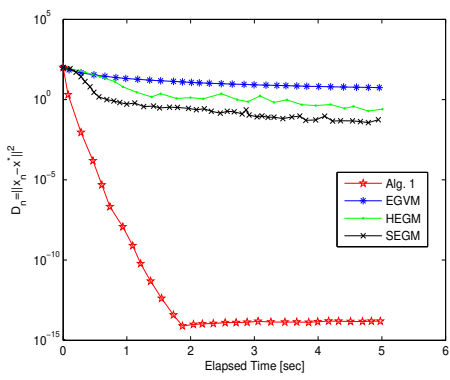

Figure 1. Experiment in $\Re^{100}$ for $\alpha_{n}=\frac{1}{\sqrt{n+1}}$

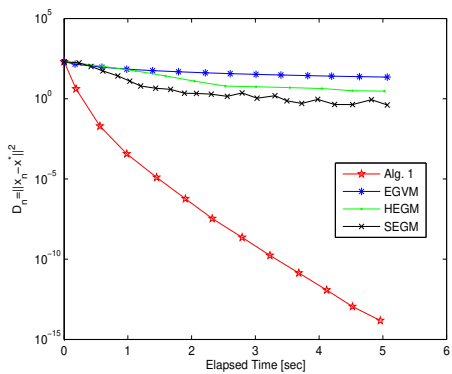

Figure 3. Experiment in $\Re^{200}$ for $\alpha_{n}=\frac{1}{\sqrt{n+1}}$

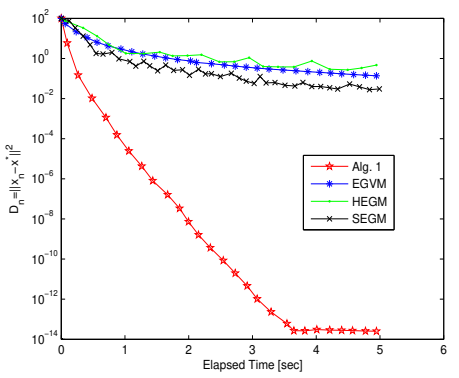

Figure 2. Experiment in $\Re^{100}$ for $\alpha_{n}=\frac{1}{n+1}$

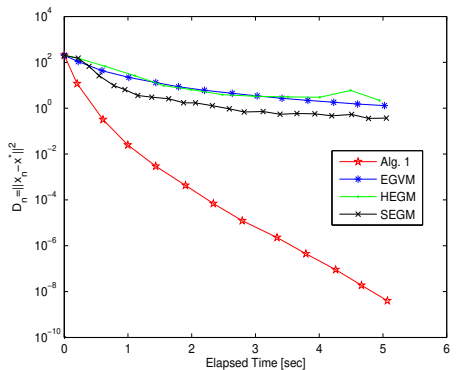

Figure 4. Experiment in $\Re^{200}$ for $\alpha_{n}=\frac{1}{n+1}$

For experiments, we have chosen the functions in the following forms:

$$
\begin{aligned}
& F_{1}(t, s)=\frac{2 t s e^{t+s}}{e \sqrt{e^{2}-1}}, f_{1}(x)=\cos x, g_{1}(t)=\frac{2 t e^{t}}{e \sqrt{e^{2}-1}} \\
& F_{2}(t, s)=\frac{\sqrt{21}}{7}(t+s), f_{2}(x)=\exp \left(-x^{2}\right), g_{2}(t)=-\frac{\sqrt{21}}{7}\left(t+\frac{1}{2}\right) .
\end{aligned}
$$

Note that $g_{1}(t)$ and $g_{2}(t)$ are generated such that $x^{*}=0$ is the solution of the problem. Since the mappings $S_{i}(x)(t)=\int_{0}^{1} F_{i}(t, s) f_{i}(x(s)) d s, i=1,2$ are Fréchet differentiable and $\left\|S_{i}^{\prime}(x) h\right\| \leq\|x\|\|h\|$ for all $x, h \in H$. Thus, a straightforward computation implies that $f$ is monotone (so, pseudomonotone) and satisfies the Lipschitz-type condition with $c_{1}=c_{2}=1$, and $U$ is nonexpansive (thus, quasi-nonexpansive). All the optimization problems in the algorithms become the projections on $C$ which is inherently explicit. All integrals in (4.1), (4.2) and others are computed by the trapezoidal formula with the stepsize $\tau=0.001$. It is not easy to compute numerically in this example for the two algorithms HEGM and SEGM due to the complexity of the hybrid or shrinking step in those two algorithms. So, we only compute in comparison between the proposed algorithm and EGVM. We illustrate the results for four parameter sequences $\alpha_{n}=\frac{1}{(n+1)^{p}}, \beta_{n}=0.5\left(1-\alpha_{n}\right), p=1,0.7,0.5,0.1$ and 
two stepsizes $\lambda_{n}=\frac{1}{5 c_{1}}$ or $\lambda_{n}=\frac{1}{2.1 c_{1}}$. The starting point is $x_{0}(t)=t+\cos t$. The results are described in Figures 5 and 6 .

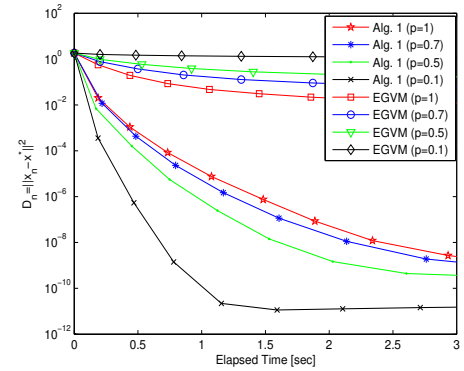

Figure 5. Experiment for stepsize $\lambda_{n}=\frac{1}{5 c_{1}}$ in Example 2

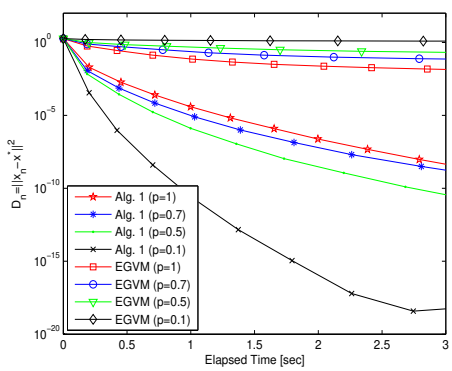

Figure 6. Experiment for stepsize $\lambda_{n}=\frac{1}{2.1 c_{1}}$ in Example 2

Example 3. In this example, we consider the problem in the Hilbert space $H=$ $l_{2}$ for the feasible set $C$ is the unit ball $B[0,1]$, the bifunction $f$ defined, for all $x=\left(x_{i}\right)_{i=1}^{\infty} \in l_{2}$ and $y=\left(y_{i}\right)_{i=1}^{\infty} \in l_{2}$, by

$$
f(x, y)=\sum_{i=1}^{\infty}\left(y_{i}^{2}+2 x_{i} y_{i}-3 x_{i}^{2}\right),
$$

and the mapping $U$ given, for all $x \in l_{2}$, by $U x=-\frac{3}{2} x$. In that case, it is easy to see that the bifunction $f$ is pseudomonotone. Moreover, $f(x, y)+f(y, z)=$ $f(x, z)-\|x-y\|^{2}-\|y-z\|^{2}+\|x-z\|^{2} \geq f(x, z)-\|x-y\|^{2}-\|y-z\|^{2}$. Thus, $f$ satisfies the Lipschitz-type condition with $c_{1}=c_{2}=1$. We see that $U$ is not nonexpansive and $F i x(U)=\{0\}$. On the other hand, we have that $\|U x-0\|^{2}=\|x-0\|^{2}+\frac{1}{5}\|(I-U) x\|^{2}$. Thus, $U$ is demicontractive with the constant $\beta=\frac{1}{5}$. We set $U_{w}=(1-w) I+w U$ with $w \in(0,1-\beta]$, and so, as mentioned in Section 2, the mapping $U_{w}$ is quasi-nonexpansive and $F i x\left(U_{w}\right)=F i x(U)$. Then, we apply Algorithm 1 for this quasi-nonexpansive mapping. Note the solution set of the problem is $x^{*}=0 \in l_{2}$. For experiment, we choose the stepsize $\lambda_{n}=\frac{2}{5}$, four parameter sequences $\alpha_{n}=\frac{1}{(n+1)^{p}}, \beta_{n}=$ $0.5\left(1-\alpha_{n}\right), p=1,0.7,0.5,0.1$, the starting point $x_{0}=\frac{\sqrt{6}}{\pi}\left(\frac{1}{i}\right)_{i=1}^{\infty}$ and the parameter $w=\frac{1}{5}$. As Example 2, we only compare Algorithm 1 and EGVM. The results are shown in Figure 7 . It is seen that the result of convergence in this example is similar to the one in Example 2.

From the aforementioned results we see that the proposed algorithm works well and its convergence is seen to be better than the one of other well known algorithms.

\section{Conclusions}

The paper has presented a new hybrid algorithm for tackling a pseudomonotone equilibrium problem with a Lipschitz-type condition, and a fixed point problem 


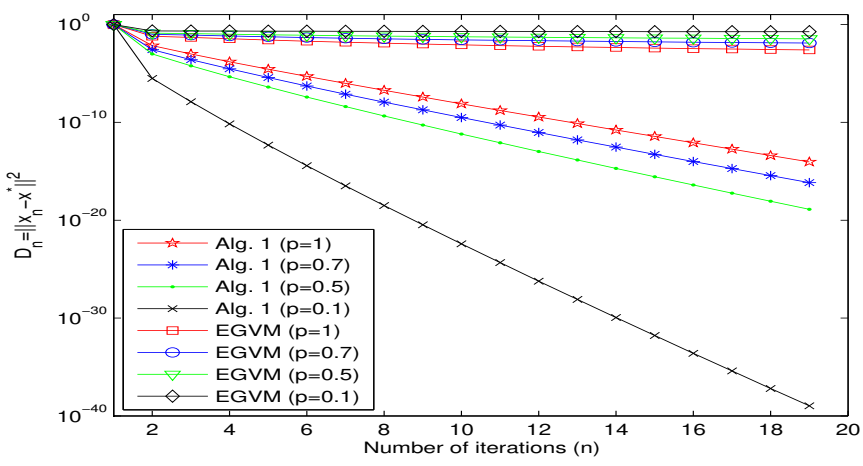

Figure 7. Experiment in Example 2

for a quasi - nonexpansive mapping in a Hilbert space. We have described how to incorporate the extragradient method (two-step proximal-like method) with a similar technique to the Mann iteration. It is known that the hybrid methods with the normal Mann iteration often have the weak convergence, while the presented algorithm has the strong convergence. We have also considered some numerical examples in both finite and infinite dimensional Hilbert spaces to illustrate the convergence and also to show the advantage of the new algorithm over existing methods in this field.

\section{References}

[1] P.N. Anh. A hybrid extragradient method extended to fixed point problems and equilibrium problems. Optimization, 62(2):271-283, 2013. https://doi.org/10.1080/02331934.2011.607497.

[2] A.S. Antipin. On convergence of proximal methods to fixed points of extremal mappings and estimates of their rate of convergence. Comp. Maths. Math. Phys., 35:539-551, 1995.

[3] H.H. Bauschke and J.A. Chen. Projection method for approximating fixed points of quasi - nonexpansive mappings without the usual demiclosedness condition. J. Nonlinear Convex Anal., 15:129-135, 2014.

[4] H.H. Bauschke and P.L. Combettes. Combettes. Convex analysis and monotone operator theory in Hilbert Spaces. New York: Springer, 2001.

[5] E. Blum and W. Oettli. From optimization and variational inequalities to equilibrium problems. Math. Student, 63:123-145, 1994.

[6] P L. Combettes and S.A. Hirstoaga. Equilibrium programming in Hilbert spaces. J. Nonlinear Convex Anal., 6:117-136, 2005.

[7] Y. Dang and Y. Gao. The strong convergence of a KM-CQ-like algorithm for a split feasibility problem. Inverse Problems, 27(1):015007, 2011. https://doi.org/10.1088/0266-5611/27/1/015007.

[8] F. Facchinei and J.S. Pang. Finite-dimensional variational inequalities and complementarity problems. Berlin: Springer, 2002. 
[9] K. Fan. A minimax inequality and applications. In: Shisha, O. (ed.) Inequality, III, Academic Press, New York, pp. 103-113, 1972.

[10] S.D. Flam and A.S. Antipin. Equilibrium programming and proximal-like algorithms. Math. Program., 78:29-41, 1997. https://doi.org/10.1007/BF02614504.

[11] D.V. Hieu. An extension of hybrid method without extrapolation step to equilibrium problems. J. Ind. Manag. Optim., 13:1723-1741, 2017.

[12] D.V. Hieu. Hybrid projection methods for equilibrium problems with nonLipschitz type bifunctions. Math. Meth. Appl. Sci., 40:4065-4079, 2017. https://doi.org/10.1002/mma.4286.

[13] D.V. Hieu. New subgradient extragradient methods for common solutions to equilibrium problems. Comput. Optim. Appl., 67(3):571-594, 2017. https://doi.org/10.1007/s10589-017-9899-4.

[14] D.V. Hieu. Convergence analysis of a new algorithm for strongly pseudomontone equilibrium problems. Numer. Algorithms., 77(4):983-1001, 2018. https://doi.org/10.1007/s11075-017-0350-9.

[15] D.V. Hieu, L.D. Muu and P.K. Anh. Parallel hybrid extragradient methods for pseudomonotone equilibrium problems and nonexpansive mappings. Numer. Algorithms., 73(1):197-217, 2016. https://doi.org/10.1007/s11075-015-0092-5.

[16] H. Iiduka and I. Yamada. A subgradient-type method for the equilibrium problem over the fixed point set and its applications. Optimization, 58(2):251-261, 2009. https://doi.org/10.1080/02331930701762829.

[17] I.V. Konnov. Application of the proximal point method to nonmonotone equilibrium problems. Journal of Optimization Theory and Applications, 119(2):317333, 2003. https://doi.org/10.1023/B:JOTA.0000005448.12716.24.

[18] I.V. Konnov. Equilibrium models and variational inequalities. Amsterdam: Elsevier, 2007.

[19] G.M. Korpelevich. The extragradient method for finding saddle points and other problems. Ekonomikai Matematicheskie Metody, 12:747-756, 1976.

[20] P. Kumam and P. Katchang. A viscosity of extragradient approximation method for finding equilibrium problems, variational inequalities and fixed point problems for nonexpansive mappings. Nonlinear Analysis: Hybrid Systems, 3(4):475486, 2009. https://doi.org/10.1016/j.nahs.2009.03.006.

[21] W. Kumam, H. Piri and P. Kumam. Solutions of system of equilibrium and variational inequality problems on fixed points of infinite family of nonexpansive mappings. Applied Mathematics and Computation, 248(1):441-455, 2014. https://doi.org/10.1016/j.amc.2014.09.118.

[22] M. Li and Y. Yao. Strong convergence of an iterative algorithm for $\lambda$-strictly pseudo-contractive mappings in hilbert spaces. An. St. Univ. Ovidius Constanta., 18(1):219-228, 2010.

[23] S.I. Lyashko and V.V. Semenov. A new two-step proximal algorithm of solving the problem of equilibrium programming. Optimization and its applications in control and data sciences. Springer, Switzerland, 115:315-325, 2016.

[24] P.E. Maingé. A hybrid extragradient-viscosity method for monotone operators and fixed point problems. SIAM J. Control Optim., 47(3):1499-1515, 2008. https://doi.org/10.1137/060675319. 
[25] P.E. Maingé and A. Moudafi. Coupling viscosity methods with the extragradient algorithm for solving equilibrium problems. J. Nonlinear Convex Anal., 9:283294, 2008.

[26] G. Mastroeni. On auxiliary principle for equilibrium problems. Publicatione del Dipartimento di Mathematica dell, Universita di Pisa, 3:1244-1258, 2000.

[27] G. Mastroeni. Gap function for equilibrium problems. J. Global. Optim., 27(4):411-426, 2003. https://doi.org/10.1023/A:1026050425030.

[28] A. Moudafi. Proximal point algorithm extended to equilibrum problem. J. Nat. Geometry, 15:91-100, 1999.

[29] A. Moudafi. Viscosity approximation methods for fixed point problems. J. Math. Anal. Appl., 241(1):46-55, 2000. https://doi.org/10.1006/jmaa.1999.6615.

[30] L.D. Muu and W. Oettli. Convergence of an adative penalty scheme for finding constrained equilibria. Nonlinear Anal. TMA, 18(2):1159-1166, 1992. https://doi.org/10.1016/0362-546X(92)90159-C.

[31] T.T.V. Nguyen, J.J. Strodiot and V.H. Nguyen. Hybrid methods for solving simultaneously an equilibrium problem and countably many fixed point problems in a Hilbert space. J. Optim. Theory Appl., 160(3):809-831, 2014. https://doi.org/10.1007/s10957-013-0400-y.

[32] T.D. Quoc, L.D. Muu and V.H. Nguyen. Extragradient algorithms extended to equilibrium problems. Optimization, 57(6):749-776, 2008. https://doi.org/10.1080/02331930601122876.

[33] S. Reich. Constructive techniques for accretive and monotone operators. Applied Nonlinear Analysis, Academic Press, New York, pp. 335-345, 1979.

[34] J.J. Strodiot, P.T. Vuong and T.T.V. Nguyen. A class of shrinking projection extragradient methods for solving non-monotone equilibrium problems in hilbert spaces. Journal of Global Optimization, 64(1):159-178, 2016. https://doi.org/10.1007/s10898-015-0365-5.

[35] S. Takahashi and W. Takahashi. Viscosity approximation methods for equilibrium problems and fixed point problems in hilbert spaces. Journal of Mathematical Analysis and Applications, 331(1):506-515, 2007. https://doi.org/10.1016/j.jmaa.2006.08.036.

[36] P.T. Vuong, J.J. Strodiot and V.H. Nguyen. Extragradient methods and linesearch algorithms for solving Ky Fan inequalities and fixed point problems. Journal of Optimization Theory and Applications, 155(2):605-627, 2012. https://doi.org/10.1007/s10957-012-0085-7.

[37] P.T. Vuong, J.J. Strodiot and V.H. Nguyen. On extragradient-viscosity methods for solving equilibrium and fixed point problems in a hilbert space. Optimization, 64(2):429-451, 2015. https://doi.org/10.1080/02331934.2012.759327.

[38] H. K. Xu. Iterative algorithms for nonlinear operators. Journal of London Mathematical Society, 66(1):240-256, 2002. https://doi.org/10.1112/S0024610702003332.

[39] I. Yamada and N. Ogura. Hybrid steepest descent method for the variational inequality problem over the the fixed point set of certain quasi-nonexpansive mappings. Numerical Functional Analysis and Optimization, 25(7-8):619-655, 2004. https://doi.org/10.1081/NFA-200045815. 